\title{
Resection of pituitary macroadenomas via the pseudocapsule along the posterior tumor margin: a cohort study and technical note
}

\author{
Davis G. Taylor, MD, John A. Jane Jr., MD, and Edward H. Oldfield, MD \\ Department of Neurological Surgery, University of Virginia Health System, Charlottesville, Virginia
}

OBJECTIVE Extracapsular resection of pituitary microadenomas improves remission rates, but the application of pseudocapsular techniques for macroadenomas has not been well described. In larger tumors, the extremely thin, compressed normal gland or its complete absence along the tumor's anterior surface limits the application of the traditional pseudocapsular technique that can be used for microadenomas. However, in the authors' experience, the interface between the pseudocapsule at the posterior margin of the adenoma and the compressed normal gland behind it is universally present, providing a surgical dissection plane. In mid-2010, the authors began using a new surgical technique to identify and use this interface for the resection of larger macroadenomas, a technique that can be used with the microscope or the endoscope.

METHODS The authors performed a cohort study using prospectively collected preoperative imaging reports and operative details and retrospectively reviewed postoperative images and clinical follow-up of patients with a pituitary macroadenoma 20-40 mm in maximum diameter undergoing microscopic transsphenoidal resection. Since dissection of the tumor capsule only pertains to encapsulated tumor within the sella and not to tumor invading the cavernous sinus, assessment of tumor removal of noninvasive tumors emphasized the entire tumor, while that of invasive tumors emphasized the intrasellar component only. The incidence of residual tumor on postoperative imaging, new-onset endocrinopathy, and recovery of preoperative pituitary deficits was compared between patients who underwent surgery before (Group A) and after (Group B) implementation of the new technique.

RESULTS There were 34 consecutive patients in Group A and 74 consecutive patients in Group B. Tumors in 18 (53\%) Group A and 40 (54\%) Group B patients had no evidence of cavernous sinus invasion on MRI. Use of the posterior pseudocapsule technique reduced the incidence of intrasellar residual tumor on postoperative MRI for tumors without cavernous sinus invasion ( $39 \%$ [Group A] vs $10 \%, p<0.05)$ and in all tumors regardless of invasion $(50 \%$ vs $18 \%, p<$ 0.005). The incidence of new endocrinopathy was less likely $(25 \% \mathrm{vs} 12 \%, p=0.098)$ and the recovery of prior deficits more likely $(13 \%$ vs $27 \%, p=0.199)$ among patients treated using the pseudocapsule approach, although the differences are not statistically significant.

CONCLUSIONS Use of the posterior pseudocapsule dissection plane can enhance the resection of pituitary macroadenomas.

https://thejns.org/doi/abs/10.3171/2017.7.JNS171658

KEY WORDS cavernous sinus; CSF leak; endocrinopathy; macroadenoma; pituitary adenoma; pituitary surgery; pseudocapsule; surgical technique

$\mathrm{U}$ SE of the histological pseudocapsule in the resection of pituitary microadenomas and small macroadenomas has been previously described and demonstrated to improve the intraoperative identification and gross-total resection of these tumors. ${ }^{8,14,16}$ For larger macroadenomas, however, the application of pseudocapsular resection can be technically challenging due to the inability to anteriorly develop the surgical plane. Many surgeons, with the aid of either the endoscope or microscope, have instead traditionally used a piecemeal resection technique. Based on our experience with pseudocapsular resection of pituitary microadenomas and smaller macroadenomas, we developed a method for the resection of larger macroadenomas whereby the tumor is internally debulked and a distinct tissue plane (the pseudocapsule) is established between the tumor and the compressed normal 
pituitary gland at the tumor's posterior margin, facilitating an extracapsular resection of the adenoma. We sought to investigate whether the use of this surgical plane improves resection of larger macroadenomas and decreases the incidence of residual tumor observed on postoperative imaging.

\section{Surgical Technique}

The surgery begins with one of the conventional approaches to the anterior face of the sella using the operating microscope or the endoscope. The bone covering the anterior and inferior surfaces of the sella is removed to expose the medial portion of the anterior face of the cavernous sinus on each side and to reach the tuberculum sellae superiorly. The dura mater is opened widely while attempting to leave the anterior surface of the pituitary (the pituitary capsule) intact.

The anterior surface of the pituitary is inspected to identify any region of compressed normal gland at the periphery of the adenoma superiorly and laterally. If a thin rim of gland is identified, a very superficial incision is made through the normal gland just before the junction of the effaced normal gland and the adenoma (this incision is very superficial, as even the most superficial incision will expose the interface between the pseudocapsule of the tumor and the compressed normal gland; see Video 2). This interface is then dissected gently with the tips of the bipolar forceps or a disc dissector. In general, with large macroadenomas (> $15 \mathrm{~mm})$, this dissection can proceed only a few millimeters along the anterior margins, but often no normal gland is evident at the anterior surface of the tumor at all. In these tumors, and in tumors in which a thin layer of the compressed normal gland is identified and initially dissected, a circular incision is then made in the central portion of the anterior face of the tumor inferiorly, and the central portion of the tumor is removed while leaving a peripheral margin that is thick enough to provide adequate integrity to the dissection plane and permit the development of the pseudocapsule plane around the entire adenoma. Given that removal of the central inferior aspect of the tumor proceeds posteriorly, the surgeon seeks to identify and define the posterior margin of the tumor at the anterior surface of the posteriorly compressed normal gland and develop the distinct interface between these tissues (the pseudocapsule along the posterior edge of the tumor); it is this interface that will be used as the plane of dissection (Fig. 1A; Video 1-0:30 and Video 2-1:23).

VIDEO 1. Sublabial microscopic approach demonstrating development of the posterior pseudocapsule and resection of a macroadenoma. Copyright Davis G. Taylor. Published with permission. Click here to view.

VIDEO 2. Endoscopic endonasal approach demonstrating development of the posterior pseudocapsule and resection of a macroadenoma. Copyright Davis G. Taylor. Published with permission. Click here to view.

The pseudocapsule is then developed with precise surgical dissection, moving from smaller disc dissectors to progressively larger-diameter ring curettes. During the initial phases of the dissection, no attempt is made to remove tumor or to pull it forward. Instead, the focus is to separate as much of the posterior and posterolateral surface of the tumor from the compressed normal gland and cavernous sinus wall as possible.

A ring curette is then used to continue the sweep of the dissection laterally (at either the 9 o'clock or 3 o'clock position) and anterolaterally around the posterolateral margin of the tumor. The tip of the instrument will have a tendency to remain within the previously developed tissue plane between the tumor and the compressed normal gland. The lateral and inferior components of the tumor are then removed using ring dissection from posterior to anterior while keeping the ring in the tissue plane of dissection (Fig. 1B; Video 1-1:14 and Video 2-1:48). In most cases, the lateral dissection is initially done on the side opposite the compressed normal gland, which is usually evident after careful inspection of the MRI studies. After this, tumor along the contralateral inferolateral margin (that which is abutting the pituitary gland) is then removed.

At this stage, much of the tumor within the sella and below the superior plane of the sella has been removed. The surgeon now endeavors to define the tissue plane between the tumor and the compressed gland along the superolateral and superior margins, usually on the side to which the gland has been displaced, typically beginning with a small disc dissector and then progressing to a larger dissector. With each stroke, the sweep of the tip of the instrument extends farther rostrally and begins to turn medially along the lateral aspect of the top margin of the tumor (Fig. 1C; Video 1-2:07 and Video 2-2:25).

At this point the $\mathrm{pCO}_{2}$ is permitted to rise to $50 \mathrm{~mm}$ $\mathrm{Hg}$. After 15-30 seconds, the resultant rise in intracranial pressure begins to deliver the tumor into the sella from above. Dissection proceeds in the visible tissue plane along the superior margin of the tumor. Progressively larger ring curettes are used within the tissue plane until the dissection proceeds across the tumor to the opposite side. By this stage, the distinct tissue plane between the surface of the tumor and the compressed normal gland against the diaphragma sellae is visible, and the dissection can often be done under direct vision with the tips of the bipolar forceps or a disc dissector (Fig. 1D). In larger tumors, the anesthesiologist may provide forced inspiration to $40 \mathrm{~mm}$ $\mathrm{Hg}$ to inferiorly displace any suprasellar component for final dissection of the superior margin of the tumor and the final stages of tumor removal. Closure is performed according to the surgeon's general preference. Application and utilization of this technique is demonstrated using either the microscope (Video 1) or endoscope (Video 2).

\section{Methods}

After receiving IRB approval, we collected the data for this study from prospectively entered presurgical imaging and operative data and retrospectively reviewed postoperative imaging and endocrine evaluation of all patients who, between 2008 and 2015, underwent microscopic sublabial, transsphenoidal resection of a pituitary adenoma performed by a single neurosurgeon (E.H.O.). Inclusion criteria required noncystic pituitary adenomas measuring $20-40 \mathrm{~mm}$ in largest diameter $(\mathrm{n}=118)$. Patients were excluded if their preoperative or postoperative imaging records were unavailable $(n=10)$. Following application 


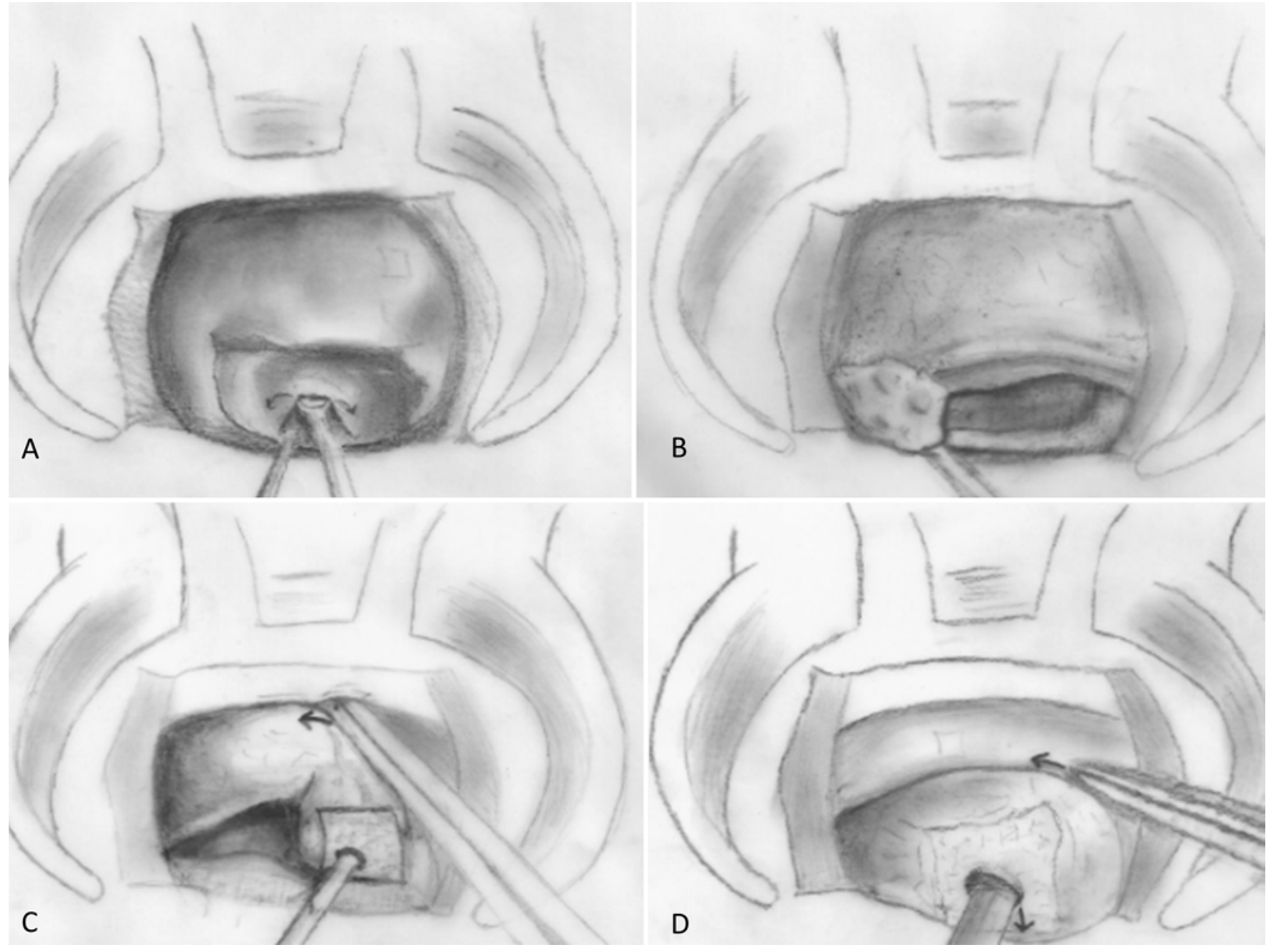

FIG. 1. A: The posterior pseudocapsule is established at the interface between the posterior aspect of the tumor and the posteriorly displaced normal gland. B: Once the plane is established, the lateral margins of the tumor are followed within this plane to deliver the tumor toward the surgeon. $\mathbf{C}$ and $\mathbf{D}$ : The plane is then developed rostrally $(\mathrm{C})$ until the interface can be directly visualized and the remaining tumor is resected (D). Copyright Davis G. Taylor. Published with permission.

of inclusion and exclusion criteria, 108 patients were available for analysis.

Preoperative images were reviewed prior to surgery by the senior author (E.H.O.) for evidence of cavernous sinus invasion, and the tumors were prospectively classified as intrasellar or invasive based on his interpretation. Surgical reports detailing the estimated extent of resection, presence of cavernous sinus invasion identified at surgery, and occurrence of intraoperative CSF leakage were prospectively entered into a database by the senior author shortly after surgery.

In this study, patients were classified according to operative technique. Patients in Group A underwent resection of a macroadenoma before introduction of the pseudocapsule technique (before July 2010). Patients in Group $\mathrm{B}$ underwent resection via the posterior pseudocapsule technique (July 2010 to November 2015).

A chart review of postoperative radiology reports and endocrine follow-up was then performed to determine the presence and location of any residual tumor at 2 months, the incidence of new-onset endocrine deficits, and the recovery of prior pituitary deficiencies at the last follow-up. We reviewed images obtained in all patients with residual tumor to determine the location (intrasellar or within the cavernous sinus) of the residual tumor. Images were then retrospectively reviewed by the first author (D.G.T.) to determine Knosp classification, ${ }^{11}$ and tumors were classified accordingly.

New pituitary deficits were determined based on 1) new laboratory deficiencies or 2) treatment with new supplemental medications without previously documented deficiencies or supplemental medications. Patients with preoperative panhypopituitarism $(n=11)$ were excluded from evaluation of new-onset deficits after surgery.

Patients with a prior abnormal pituitary deficit that returned to normal during follow-up or those previously receiving supplemental medications that were subsequently discontinued at follow-up were considered to have recovered from a preoperative pituitary deficiency. Patients without preoperative endocrine deficits $(n=34)$ were ex- 
cluded from analysis of the recovery of deficits. Two patients were not included in analysis of endocrine recovery: one due to inadequate laboratory follow-up and the other due to concurrent androgen suppression treatment for prostate cancer in the setting of a testosterone deficiency.

The presence of residual tumor on postoperative MRI, the extent of resection estimated at surgery, intraoperative CSF leakage, and new-onset endocrine deficits were compared between Groups A and B in patients with and without cavernous sinus invasion. Statistical analysis was performed using the chi-square test for categorical outcomes.

\section{Results}

There were 34 patients in Group A and 74 in Group B. Tumors in 16 (47\%) Group A and 34 (46\%) Group B patients had evidence of cavernous sinus invasion on preoperative MRI ( $\mathrm{p}=0.874)$. Fourteen $(44 \%)$ Group A patients and $25(35 \%)$ Group B patients had Knosp grades of 3 or $4(\mathrm{p}=0.413)$. At surgery, $12(38 \%)$ Group A and $38(51 \%)$ Group B patients had evidence of cavernous sinus invasion $(p=0.152)$. There were no differences in the incidence of prior surgery $(p=0.1)$, time to imaging follow-up $(p=0.9)$, time to endocrine follow-up $(\mathrm{p}=0.5)$, size of tumor $(\mathrm{p}=$ $0.3)$, incidence of preoperative endocrine deficits (0.896), or tumor type (Table 1).

Among tumors without evidence of cavernous sinus invasion on prospective preoperative MRI evaluation or those with retrospective Knosp grades of 1 or 2, the incidence of residual tumor on postoperative imaging was reduced when the posterior pseudocapsular technique (Group B) was used (10\% [Group B] vs 39\%, p < 0.05; and $15 \%$ [Group B] vs 39\%, p < 0.05, respectively). Moreover, when there was no evidence of cavernous sinus invasion at surgery, residual tumor was again less likely among Group B patients $(9 \%$ vs $50 \%, \mathrm{p}<0.05)$. Furthermore, when limited to the intrasellar component of all tumors (including those with cavernous sinus invasion), use of the posterior pseudocapsule technique resulted in reduced incidence of residual tumor (18\% [Group B] vs 50\%, p < 0.005) (Table 2).

There was a trend toward significance in the rate of postoperative endocrine deficits between Group A and Group B patients ( $25 \%$ vs $12 \%, \mathrm{p}=0.098)$, but this was not statistically significant. There was no difference in the rate of recovery of previous deficits $(13 \%$ vs $27 \%$, p $=0.199)$. Intraoperative CSF leakage was less likely in Group B patients (22\% vs 38\%, p = 0.07), but use of an intraoperative lumbar drain was more frequent in this group ( $73 \%$ vs $30 \%, p<0.005$ ) (Table 1). When controlling for use of intraoperative lumbar drain, there was no difference between techniques in the rate of intraoperative CSF leakage among patients with $(0 \%$ vs $17 \%, \mathrm{p}=0.164)$ and without (54\% vs $35 \%, \mathrm{p}=0.204)$ an intraoperative lumbar drain (Table 3).

\section{Discussion}

Pituitary adenomas arise from a single cell within the anterior pituitary. While the adenoma lacks the typical reticulin-supported acinar structure of the normal gland
TABLE 1. Univariate analysis of demographic data of Group A and Group B patients

\begin{tabular}{lcccc}
\hline \multicolumn{1}{c}{ Demographics } & Total & Group & Group & p \\
Value
\end{tabular}

$\mathrm{CSI}=$ cavernous sinus invasion; $\mathrm{DI}$ = diabetes insipidus; $\mathrm{GH}=$ growth hormone; NFA = nonfunctioning adenoma; $\mathrm{TSH}=$ thyroid-stimulating hormone.

* Data were missing for 4 patients (2 patients in each group).

(contributing to the tumor's often suckable and soft nature), tumor growth leads to compression of the adjacent normal gland and its acinar structure and reticulin microskeleton, resulting in a surgical pseudocapsule that encases the entire adenoma and separates it from the normal gland.

In 1936, Costello demonstrated the presence of 225 adenomas among 1000 pituitary glands examined at autopsy. ${ }^{5}$ Many of the tumors he described were notable for a boundary of a thin layer of compressed reticulin, the histological pseudocapsule, which was quite distinct from the pituitary capsule derived from the pia mater as 
TABLE 2. Univariate analysis of residual tumor

\begin{tabular}{|c|c|c|c|c|}
\hline Variable & Total & Group A & Group B & p Value \\
\hline \multicolumn{5}{|c|}{ Residual intrasellar tumor based on technique } \\
\hline All tumors & $30(28 \%)$ & $17(50 \%)$ & $13(18 \%)$ & $<0.005$ \\
\hline w/o CSI on preop imaging* & $11(19 \%)$ & $7(39 \%)$ & $4(10 \%)$ & 0.009 \\
\hline w/o CSI at op $\dagger$ & $13(24 \%)$ & $10(50 \%)$ & $3(9 \%)$ & $<0.005$ \\
\hline w/o CSI at op \& w/ presumed GTR & $10(20 \%)$ & $7(41 \%)$ & $3(9 \%)$ & 0.007 \\
\hline \multicolumn{5}{|l|}{ Knosp grade $\ddagger$} \\
\hline 1 or 2 & $14(22 \%)$ & $7(39 \%)$ & $7(15 \%)$ & 0.039 \\
\hline 3 or 4 & $15(38 \%)$ & $10(71 \%)$ & $5(20 \%)$ & $<0.05$ \\
\hline \multicolumn{5}{|l|}{ Any residual tumor based on technique } \\
\hline All tumors & $44(41 \%)$ & $19(56 \%)$ & $25(34 \%)$ & 0.323 \\
\hline w/o CSI on preop imaging* & $12(21 \%)$ & $7(39 \%)$ & $5(13 \%)$ & 0.022 \\
\hline w/o CSI at op $\dagger$ & $13(24 \%)$ & $10(50 \%)$ & $3(9 \%)$ & $<0.005$ \\
\hline w/o CSI at op \& w/ presumed GTR & $10(20 \%)$ & $7(41 \%)$ & $3(9 \%)$ & 0.007 \\
\hline \multicolumn{5}{|l|}{ Knosp grade } \\
\hline 1 or 2 & $17(27 \%)$ & $7(39 \%)$ & $10(22 \%)$ & 0.16 \\
\hline 3 or 4 & $26(67 \%)$ & $12(86 \%)$ & $14(56 \%)$ & 0.06 \\
\hline
\end{tabular}

TABLE 3. Univariate analysis of intraoperative CSF leak, postoperative endocrine deficits, and recovery of prior deficits

\begin{tabular}{|c|c|c|c|c|c|}
\hline Variable & No. of Patients* & Total & Group A & Group B & $\mathrm{p}$ Value \\
\hline \multicolumn{6}{|l|}{ Rate of endocrine deficits based on technique } \\
\hline All patients w/ preop pituitary function & $97,28,69$ & $15(15 \%)$ & $7(25 \%)$ & $8(12 \%)$ & 0.098 \\
\hline w/o CSI on preop imaging & $50,14,36$ & $6(12 \%)$ & $2(14 \%)$ & $4(11 \%)$ & 0.756 \\
\hline w/o CSI at op & $48,16,32$ & $4(8 \%)$ & $1(6 \%)$ & $3(9 \%)$ & 0.712 \\
\hline \multicolumn{6}{|l|}{ Rate of recovery of prior deficits } \\
\hline All patients w/ preop deficit & $72,23,49$ & $16(22 \%)$ & $3(13 \%)$ & $13(27 \%)$ & 0.199 \\
\hline w/o CSI on preop imaging & $45,14,31$ & $11(24 \%)$ & $3(21 \%)$ & $8(26 \%)$ & 0.752 \\
\hline w/o CSI at op & $39,16,23$ & $10(26 \%)$ & $3(19 \%)$ & $7(30 \%)$ & 0.411 \\
\hline \multicolumn{6}{|l|}{ Rate of CSF leak based on technique } \\
\hline All tumors & $108,34,74$ & $29(27 \%)$ & $13(38 \%)$ & $16(22 \%)$ & 0.070 \\
\hline When intraop lumbar drain used & $64,10,54$ & $9(14 \%)$ & $0(0 \%)$ & $9(17 \%)$ & 0.164 \\
\hline When no intraop lumbar drain used & $44,24,20$ & $20(45 \%)$ & $13(54 \%)$ & $7(35 \%)$ & 0.204 \\
\hline
\end{tabular}

* Presented as total, Group A, and Group B patients, respectively.

described by Ciric. ${ }^{3}$ In the mid-1980s, the senior author (E.H.O.) consistently began removing adenomas using the dissection of the pseudocapsule as a surgical capsule, and, in 2005, Kawamata et al. described the importance of including the pseudocapsule in the excision of growth hormone-secreting tumors for improved endocrine remission of growth hormone-producing tumors. ${ }^{9}, 16$

Use of the histological pseudocapsule as a surgical capsule has been previously demonstrated to improve grosstotal resection, remission rates, and rate of tumor recurrence, as well as to improve the resolution of preoperative endocrinopathies among microadenomas. ${ }^{8,9,14,18}$ Utilization of the pseudocapsule among macroadenomas, howev- er, has been discussed only in limited series and with little to no emphasis on surgical technique and with contrasting conclusions regarding the efficacy of pseudocapsular resection. ${ }^{12,19,23}$ Instead, traditional resection of macroadenomas, via either an endoscopic or microscopic approach, has typically used a piecemeal resection technique., ${ }^{1,6}$ The piecemeal technique, however, results in reduced visibility of the tumor margins as the tumor is centrally decompressed and the remaining tumor descends and the normal gland expands. Failure to establish a surgical dissection plane (as with the pseudocapsular technique) can obscure the tumor margins and leave behind remnants of isolated tumor, leading to overestimation of the extent of resection 
and high rates of residual tumor regardless of whether the microscope or endoscope was used. 2,4,7,13,17,20

In the current series, $10(20 \%)$ of all intrasellar tumors without evidence of cavernous sinus invasion at surgery and with presumed gross-total resection had residual tumor at follow-up. Of those, 7 tumors were in Group A and 3 were in Group B, resulting in an overestimation of extent of resection in $41 \%$ and $9 \%(\mathrm{p}<0.05)$, respectively (Table 2). These findings are consistent with those in a series by Kim et al. in which piecemeal resection was associated with a significantly lower incidence of gross-total resection than pseudocapsular resection among pituitary adenomas with a Hardy classification of less than $3 .^{10}$ As demonstrated here, development of the posterior pseudocapsule establishes a surgical plane and provides a superior opportunity for true gross-total resection and reduces the incidence of residual intrasellar tumor on postoperative imaging in the absence of cavernous sinus invasion $(39 \%$ vs $10 \%, \mathrm{p}<0.05$ based on preoperative imaging; $50 \%$ vs $9 \%, \mathrm{p}<0.005$ based on intraoperative findings); and for any location (extrasellar included) regardless of cavernous sinus invasion ( $56 \%$ vs $34 \%, \mathrm{p}<0.05$; Table 2 ).

By improving gross-total resection rates and extent of resection in tumors with cavernous sinus invasion, repeat surgery and the necessity of postoperative radiation therapy for residual tumor may be avoided, while more complete resection results in improved tumor growth control rates, reduces the postoperative radiation dose, and decreases the risk of cranial nerve dysfunction following postoperative Gamma Knife radiosurgery among nonfunctioning macroadenomas. ${ }^{22}$ Among functioning adenomas, reduced postoperative tumor volume may lead to more rapid resolution of endocrinopathy after radiation treatment. ${ }^{15,17,21}$

In our study, the incidence of new endocrine deficits was much less likely among Group B patients (25\% vs $12 \%)$ with a strong trend toward significance $(\mathrm{p}=0.098)$, while the rate of recovery of prior deficits was similar between groups ( $13 \%$ vs $27 \%, \mathrm{p}=0.199)$, indicating that the development of the posterior pseudocapsule in the resection of macroadenomas is equally as safe with respect to pituitary function as traditional techniques, and the trend toward significance indicates that the posterior pseudocapsule technique may prove to improve the rate of new endocrinopathies in a larger cohort. Combined with the reduced incidence of residual tumor on postoperative imaging, we believe that pseudocapsular resection along the posterior margin of macroadenomas should be considered by both endoscopic and microscopic pituitary surgeons.

\section{Conclusions}

Use of the posterior pseudocapsule dissection plane in macroadenomas significantly decreases the incidence of postoperative residual tumor without increasing the risk of intraoperative CSF leakage or postoperative endocrine deficits and provides a similar rate of recovery of prior endocrine deficiencies. This technique is applicable for both the endoscope and the microscope. More complete resection of adenomas may have a significant impact on postoperative management.

\section{References}

1. Bi WL, Smith TR, Nery B, Dunn IF, Laws ER: Pituitary tumors: functioning and nonfunctioning, in Winn HR (ed): Youmans \& Winn Neurological Surgery, ed 7. Philadelphia: Elsevier, 2016, pp 1155-1182

2. Bohinski RJ, Warnick RE, Gaskill-Shipley MF, Zuccarello M, van Loveren HR, Kormos DW, et al: Intraoperative magnetic resonance imaging to determine the extent of resection of pituitary macroadenomas during transsphenoidal microsurgery. Neurosurgery 49:1133-1144, 2001

3. Ciric I: On the origin and nature of the pituitary gland capsule. J Neurosurg 46:596-600, 1977

4. Ciric I, Mikhael M, Stafford T, Lawson L, Garces R: Transsphenoidal microsurgery of pituitary macroadenomas with long-term follow-up results. J Neurosurg 59:395-401, 1983

5. Costello RT: Subclinical adenoma of the pituitary gland. Am J Pathol 12:205-216-1, 1936

6. Couldwell WT: Transsphenoidal and transcranial surgery for pituitary adenomas. J Neurooncol 69:237-256, 2004

7. Fahlbusch R, Ganslandt O, Buchfelder M, Schott W, Nimsky $\mathrm{C}$ : Intraoperative magnetic resonance imaging during transsphenoidal surgery. J Neurosurg 95:381-390, 2001

8. Jagannathan J, Smith R, DeVroom HL, Vortmeyer AO, Stratakis CA, Nieman LK, et al: Outcome of using the histological pseudocapsule as a surgical capsule in Cushing disease. J Neurosurg 111:531-539, 2009

9. Kawamata T, Kubo O, Hori T: Surgical removal of growth hormone-secreting pituitary adenomas with intensive microsurgical pseudocapsule resection results in complete remission of acromegaly. Neurosurg Rev 28:201-208, 2005

10. Kim EH, Ku CR, Lee EJ, Kim SH: Extracapsular en bloc resection in pituitary adenoma surgery. Pituitary 18:397-404, 2015

11. Knosp E, Steiner E, Kitz K, Matula C: Pituitary adenomas with invasion of the cavernous sinus space: a magnetic resonance imaging classification compared with surgical findings. Neurosurgery 33:610-618, 1993

12. Lee EJ, Ahn JY, Noh T, Kim SH, Kim TS, Kim SH: Tumor tissue identification in the pseudocapsule of pituitary adenoma: should the pseudocapsule be removed for total resection of pituitary adenoma? Neurosurgery 64 (3 Suppl):ons62ons70, 2009

13. Losa M, Mortini P, Barzaghi R, Ribotto P, Terreni MR, Marzoli SB, et al: Early results of surgery in patients with nonfunctioning pituitary adenoma and analysis of the risk of tumor recurrence. J Neurosurg 108:525-532, 2008

14. Monteith SJ, Starke RM, Jane JA Jr, Oldfield EH: Use of the histological pseudocapsule in surgery for Cushing disease: rapid postoperative cortisol decline predicting complete tumor resection. J Neurosurg 116:721-727, 2012

15. Oldfield EH, Jane JA Jr, Thorner MO, Pledger CL, Sheehan JP, Vance ML: Correlation between GH and IGF-1 during treatment for acromegaly. J Neurosurg 126:1959-1966, 2017

16. Oldfield EH, Vortmeyer AO: Development of a histological pseudocapsule and its use as a surgical capsule in the excision of pituitary tumors. J Neurosurg 104:7-19, 2006

17. Przybylowski CJ, Dallapiazza RF, Williams BJ, Pomeraniec IJ, Xu Z, Payne SC, et al: Primary versus revision transsphenoidal resection for nonfunctioning pituitary macroadenomas: matched cohort study. J Neurosurg 126:889-896, 2017

18. Qu X, Xu G, Qu Y, Song T: The pseudocapsule surrounding a pituitary adenoma and its clinical significance. J Neurooncol 101:171-178, 2011

19. Qu X, Yang J, Sun JD, Mou CZ, Wang GD, Han T, et al: Transsphenoidal pseudocapsule-based extracapsular resection for pituitary adenomas. Acta Neurochir (Wien) 153:799-806, 2011

20. Serra C, Burkhardt JK, Esposito G, Bozinov O, Pangalu A, Valavanis A, et al: Pituitary surgery and volumetric assess- 
ment of extent of resection: a paradigm shift in the use of intraoperative magnetic resonance imaging. Neurosurg Focus 40(3):E17, 2016

21. Sheehan JP, Kondziolka D, Flickinger J, Lunsford LD: Radiosurgery for residual or recurrent nonfunctioning pituitary adenoma. J Neurosurg 97 (5 Suppl):408-414, 2002

22. Sheehan JP, Starke RM, Mathieu D, Young B, Sneed PK, Chiang VL, et al: Gamma Knife radiosurgery for the management of nonfunctioning pituitary adenomas: a multicenter study. J Neurosurg 119:446-456, 2013

23. Witek P, Zieliński G, Szamotulska K, Maksymowicz M, Kamiński G: Clinicopathological predictive factors in the early remission of corticotroph pituitary macroadenomas in a tertiary referral centre. Eur J Endocrinol 174:539-549, 2016

\section{Disclosures}

The authors report no conflict of interest concerning the materials or methods used in this study or the findings specified in this paper.

\section{Author Contributions}

Conception and design: Taylor, Oldfield. Acquisition of data: Oldfield. Analysis and interpretation of data: Taylor, Oldfield. Drafting the article: all authors. Critically revising the article: all authors. Reviewed submitted version of manuscript: all authors. Approved the final version of the manuscript on behalf of all authors: Taylor. Statistical analysis: Taylor, Oldfield. Administrative/technical/material support: all authors. Study supervision: Oldfield.

\section{Supplemental Information}

Videos

Video 1. https://vimeo.com/227250941.

Video 2. https://vimeo.com/227251037.

\section{Correspondence}

Davis G. Taylor, Department of Neurological Surgery, University of Virginia, Box 800212, Charlottesville, VA 22908. email: dt4aa@virginia.edu. 